\title{
Observation of Anderson localization of light in three dimensions
}

\author{
Christof M. Aegerter, ${ }^{*}$ Martin Störzer, Susanne Fiebig, Wolfgang Bührer, and Georg Maret \\ Fachbereich Physik, University of Konstanz, Box M621, 78457 Konstanz, Germany \\ *Corresponding author: christof.aegerter@uni-konstanz.de
}

Received October 6, 2006; revised April 4, 2007; accepted April 16, 2007;

posted April 20, 2007 (Doc. ID 75790); published August 8, 2007

\begin{abstract}
Using time-resolved transmission measurements, we have found indications of Anderson localization of light in bulk three-dimensional systems. The observed deviation from classical diffusion is in good accord with theoretical predictions of localization and cannot be explained by absorption or experimental artifacts such as stratification, fluorescence, or background illumination. Moreover, we show that in our samples the control parameter is given by the mean free path times the wavenumber as required by the Ioffe-Regel criterion. This is in contrast to quasi-one-dimensional systems that were studied with microwaves. There, the control parameter is related to the number of modes inside a waveguide, and deviations from classical diffusion are possible due to a small number of modes. (C) 2007 Optical Society of America

OCIS codes: $290.1990,260.3160,350.5500$.
\end{abstract}

\section{INTRODUCTION}

The quest for an experimental verification of Anderson localization [1] of light has been a long and controversial one. Because detailed theories for localization in finite samples are exceedingly difficult, the experimental signatures of localization are somewhat ambivalent. The initial predictions by Anderson [2] in 1985 and John [3] in 1984 of an exponentially decreased transmission in the localized state and the scaling of the diffusion coefficient with sample thickness have both been used as characteristic measures of the onset of localization $[4,5]$. Such measures concerning the average properties of photons can, however, also be obtained from effects acting on all scatterers without taking into account localization. For instance absorption can similarly lead to an exponential decrease of the total transmission of a sample [6-8]. The prediction of localization theory can be compared to measurements of total transmission only if the absorption length is known from an independent determination. Similarly, a decrease in the diffusion coefficient can be obtained by resonant scattering from particles that are of size comparable with the wave length [9]. In order to check for this, the transport speed of photons has to be determined independently.

A clearcut signal of localization thus has to take into account the properties of paths leading to localization, i.e., that they are multiple-scattering paths with a high probability of forming closed loops. This can be achieved either by time-resolved measurements (of transmission [10] or reflection [11]) or by studying the fluctuations in transmission. Both of these measures have been used to characterize the transmission of microwaves through quasi-one-dimensional, disordered waveguides [8,12]. Due to the restricted geometry of these waveguides, the control parameter that needs to be varied to observe localization is the dimensionless conductance, $g$, which is related to the number of modes in the waveguide. This is basically a measure of the inverse probability of different photon paths crossing inside the sample, which in this restricted geometry is naturally small. The fact that the dimensionless conductance is small leads to the presence of "prelocalized" states [13], where interference on multiply crossed paths is important [14]. These states then lead to deviations from classical diffusion, which have also been found in theoretical investigations of the problem [14,15].

In bulk systems, however, the control parameter of localization is given by the product of the wavenumber $k$ and the mean free path $l^{*}$ as introduced by Ioffe and Regel [16]. This parameter can be reasonably high for the quasione-dimensional samples studied in microwave experiments. This implies that in spite of the deviations from classical diffusion due to prelocalized states discussed above, the transition to localization as given by the IoffeRegel criterion has not yet been passed.

Here we present time-resolved measurements of transmission at optical wavelengths [10] of bulk samples (typical dimensions are $10^{5} \times 10^{5} \times 10^{4}$ in units of $l^{*}$ ) [17]. This implies that while $k l^{*}$ is low in our samples (varying between 2.5 and 30), the dimensionless conductance is huge with $g \simeq 10^{4}[18,19]$. Thus the situation cannot be compared to that of the quasi-one-dimensional experiments using microwaves. In our samples, any deviations from classical diffusion should not be due to prelocalized states but rather to localization on very long multiple-scattering loops intersecting with themselves. This is also in good accord with recent theories of localization in open, threedimensional media [20], where deviations from the classical picture set in only at the threshold to strong localization. The results obtained in [20] are also in qualitative agreement with our data in that samples below the localization threshold show a subexponential decrease of transmission, which sets in only at long times. For a quantitative comparison, however, this theory is not suitable, as calculations describing our very thick samples would be much too time consuming [21]. Therefore, our 
transmission spectra are described by a phenomenological theory incorporating a temporally varying diffusion coefficient [22,23], where the diffusion coefficient decreases in inverse proportion to the time after a certain time connected to the localization length [24]. Finally, we exclude a number of experimental artifacts that could produce similar deviations from a diffusive pulse.

\section{EXPERIMENTAL SETUP}

Our samples consist of commercially available powders of $\mathrm{TiO}_{2}$ with an average grain size ranging from 220 to $540 \mathrm{~nm}$ and a polydispersity of $\sim 20 \%$. These powders are usually used as the basis of pigments for white paint and are obtained from DuPont chemical company. At the wavelength of the experiment, $590 \mathrm{~nm}, \mathrm{TiO}_{2}$ has a high refractive index of 2.73 , such that these samples have a very small transport mean free path $l^{*}$. To achieve this, the powders are packed between two glass plates to a packing fraction of $\sim 40-45 \%$ [17].

In order to experimentally determine the turbidity of the samples, the samples are characterized by coherent backscattering. Due to weak localization effects, the reflected light shows a twofold enhancement in the backdirection $[25,26]$. This enhancement decays back to the incoherent background over a scale given by the inverse of $k l^{*}$ [27], thus providing a direct measure of the turbidity. In strongly scattering samples, such as ours, the effective refractive index of the medium [28] has to be taken into account as well. Due to the jump in refractive index at the sample boundary, reflections occur, which lead to a narrowing of the enhancement. This correction has been calculated [29], and leads to a shift in the values of $k l^{*}$ obtained from backscattering measurements of roughly a factor of two depending on the packing fraction of the sample. In order to carry out this correction, the effective refractive index has to be known, which we calculate from the energy coherent potential approximation [30] given the size and packing fraction of each sample. The angleresolved intensity was measured using a custom designed setup consisting of 256 sensitive photodiodes placed at fixed angles in an arc of $1.2 \mathrm{~m}$ diameter. The placement of the photodiodes is such that in the central part $\left(\theta<10^{\circ}\right)$ the resolution is highest $\left(0.15^{\circ}\right)$ and decreases toward the outer angles. Therefore the setup allows a study of angles up to $85^{\circ}[31,32]$.

After the samples' turbidity has been characterized, we measure their time-resolved transmission. This is done using a pulsed dye laser capable of delivering pulses of a width of $\sim 20 \mathrm{ps}$ at a repetition rate of a few megahertz. Due to the dye used (Rhodamin 6G), we are able to change the lasing wavelength continuously between 570 and $620 \mathrm{~nm}$. In the following, however, the wavelength is kept constant at $590 \mathrm{~nm}$ [17]. The time-of-flight spectra of the different samples are then obtained by passing a pulse through the sample after which it is recorded by a photomultiplier (PM). The signal from the PM starts a clock, which is subsequently stopped by the signal from a delayed reference pulse that has been extracted from the pulse just before the sample. A histogram of many of these time differences then gives a determination of the timeof-flight distribution, which is directly related to the path- length distribution of diffusive paths through the samples, which can be calculated analytically [10]. At long times, this yields an exponential decrease of the transmission

$$
T(t) \propto\left(\frac{D(t)}{D_{0}}\right)^{2} \exp \left[-\left(\frac{\pi^{2} D(t)}{L^{2}}+\frac{1}{\tau_{a b s}}\right) t\right],
$$

where the characteristic time scale is given by the thickness $L$, the diffusion coefficient $D$, and the absorption time $\tau_{a b s}$.

Due to the presence of afterpulses, a nonsymmetric pulse shape, electronic broadening, and background noise in the system response, the time-of-flight distributions have to be deconvoluted by the system response in the absence of a sample. The results thus obtained correspond directly to the calculated path-length distribution that assume an incident delta-shaped pulse.

The simultaneous measurement of $k l^{*}$ using coherent backscattering and the diffusion coefficient $D$ from timeof-flight measurements also allows an experimental determination of the transport velocity $v_{T}$. Thus a test of the influence of resonance scattering on the results is possible as well [33].

\section{RESULTS}

In contrast to the theoretical prediction of Eq. (1) for classical diffusion, the time-of-flight distributions for a sample with $k l^{*}=2.5$ shows a clearly nonexponential decay at long times; see Fig. 1 . While this is consistent with a time dependence of $D$ as discussed below, some properties of the experiment might also give rise to a long-time tail. Such artifacts have to be excluded before discussing the implications of the data on localization. $\mathrm{TiO}_{2}$ is known to show fluorescence when strongly compressed [34]. Thus the small amount of photons in this tail might arise from fluorescence. We have checked for this by placing a filter suppressing wavelengths higher than $590 \mathrm{~nm}$ between

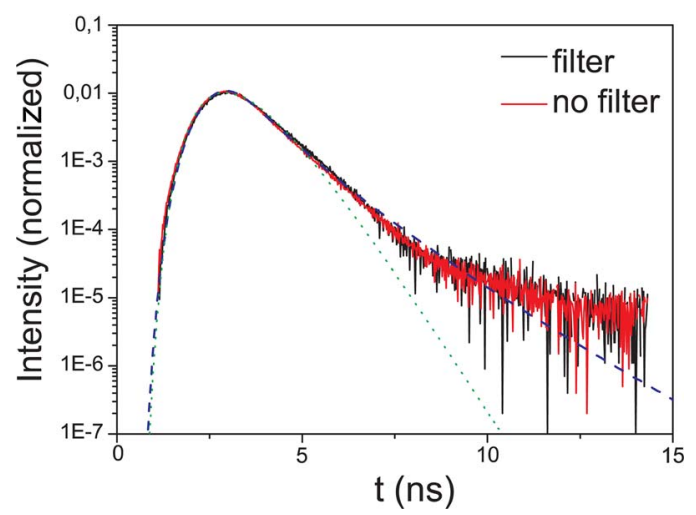

Fig. 1. (Color online) Time resolved transmission through a localizing sample. The onset of localization can be seen from the nonexponential tail in the distribution at long times giving rise to deviations from classical diffusion (dotted curve). This tail is not due to fluorescence of the sample, as can be checked by placing a filter behind the sample that suppresses photons of wavelength higher than that of the illuminating laser. Moreover, the tail is well described by a time-dependent diffusion coefficient, decreasing with time as $1 / t$, as required for localization (dashed curve). 
the sample and the PM. The result of this is shown by the black curve in Fig. 1, which is indistinguishable from the result without the presence of a filter. Fluorescence can therefore be excluded. On the other hand, a layering in the sample preparation might lead to a distribution of values of $l^{*}$ through the sample. This in turn would give rise to a nonexponential decay of the time-resolved transmission. In order to check for this, we have illuminated the sample from the front and the back, respectively. If the nonexponential decay observed in front illumination (see Fig. 1) were due to such layering, a flipped sample would give rise to a faster than exponential decrease. Both types of illumination, however, give the same result, thus ruling out layering as the source of the nonexponential decay. Another difficulty might be the presence of background illumination, which starts to be important in turbid samples, where transmitted intensity is decreased. However, when we studied the same sample at different thicknesses, such that the transmitted intensity was changed by a factor of ten, no difference in the long-time tail was observed. This implies that background illumination does not play a role in the deviation from classical diffusion in the turbid samples. In addition, a reduction in transport speed due to resonance scattering has been shown to influence the determination of $D$ from time-offlight measurements [5,9]. However, in our case, we directly determine $v_{T}$ as discussed above, which shows that for the sample with the strongest deviation from classical diffusion, $v_{T}$ is well described by the effective refractive index [33]. Finally, absorption needs to be treated carefully when one is studying localization effects. However, absorption would only lead to an additional exponential decrease of the time-resolved transmission and could not explain the nonexponential tail. In addition, we can determine the absorption length $L_{a}=\sqrt{D \tau_{a b s}}$, which does not show a systematic dependence on $k l^{*}$, as shown in Fig. 2.

The deviations from classical diffusion, in contrast, show a strong increase with decreasing $k l^{*}$, indicating a

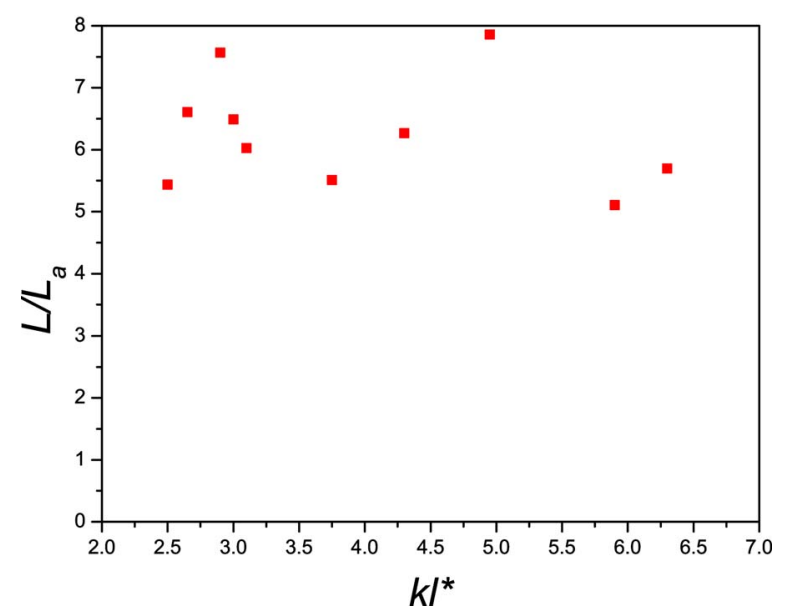

Fig. 2. (Color online) The inverse absorption length, $L_{a}$, does not show a systematic dependence on $k l^{*}$, in contrast to the localization length. This shows that the deviations from classical diffusion are not associated with absorption. However, the fits to the time-resolved transmission data make an explicit determination of the absorption length possible, such that the exponential decay of the total transmission can be compared with that predicted solely from absorption.

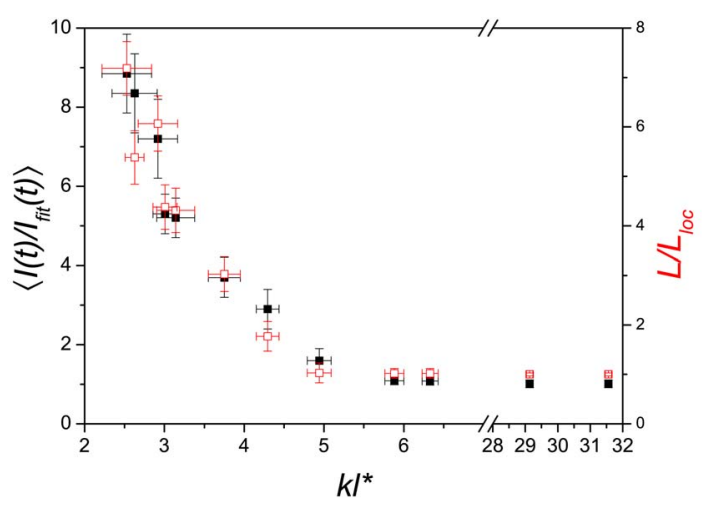

Fig. 3. (Color online) Inverse localization length as a function of the control parameter $k l^{*}$ (open symbols) [24]. This physical measure is in good agreement with an empirical measure of the deviation of the data from classical diffusion, which can be obtained from averaging the ratio of the data to a classical fit over a range from $t_{\max }$ to $3 t_{\max }$ (solid symbols) [17].

phase transition as required by the Ioffe-Regel criterion [16]. This can be seen in Fig. 3, where we show the average of the ratio of the data to a fit using classical diffusion theory (dotted curve in Fig. 1) as a function of $k l^{*}$. This shows a marked increase starting at $k l^{*}$ values around 4-5. In addition, the figure shows a determination of the localization length, $L_{l o c}$, which shows the same qualitative behavior. The localization length is determined from a fit to the data using a temporally varying diffusion coefficient [22] (dashed curve in Fig. 1). As has also been found by simulations [35], in the case of localization the mean square displacement, $\left\langle r^{2}\right\rangle$, of photons saturates after a characteristic time, such that the effective $D$ becomes proportional to $1 / t$. This has been added to the behavior in Eq. (1), where the localization length is determined by the diffusion coefficient at early times and the time scale where the time dependence sets in, $\tau_{l o c}$, i.e. $L_{l o c}=\sqrt{D \tau_{l o c}}$ [24]. In order to capture the behavior of both classical diffusion $\left(\left\langle r^{2}\right\rangle \propto t\right)$ and localized states $\left(\left\langle r^{2}\right\rangle\right.$ =const.), we have fitted the data with a mean square displacement varying as $t^{-\alpha}$, where the exponent $\alpha$ is a fitting parameter [24]. The resulting exponent for all of the samples investigated is shown in Fig. 4. As can be seen, the behavior changes from classical at high $k l^{*}$ (with $\alpha=$ 1) to localized at $k l^{*}$ below 4 (with $\alpha=0$ ). Right at the transition, it can even be seen that the prediction from classical scaling theory $[21,36]$ of $\alpha=2 / 3$ is consistent with the data.

Finally, we have performed measurements of static transmissions on our most localizing sample as well as on a classical sample. The result of this is shown in Fig. 5, where a nearly exponential decrease of the transmitted intensity can be seen in both cases. Indeed, localization theory predicts an exponential decrease of the static transmission in the localized state, in contrast to the $1 / L$ dependence of a purely diffusive sample (dotted curves in Fig. 5). However, this measurement alone cannot be seen as an indication of localization in our sample, since absorption will similarly lead to an exponential decrease in intensity. However, there will be a difference in the char- 


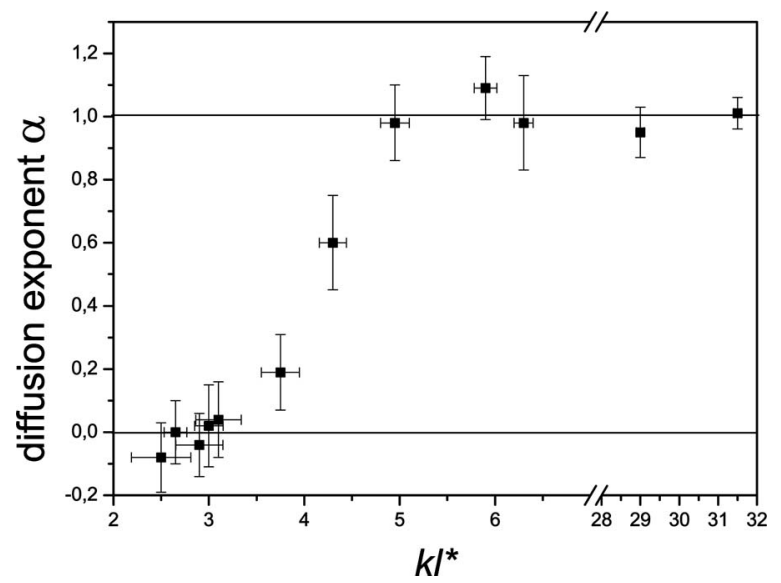

Fig. 4. The time dependence of the width of the photon cloud can be described by an exponent, $\alpha$, which is unity for classical diffusion and zero in the case of localization [23]. Just at the transition, a value of $\alpha=2 / 3$ is predicted from one-parameter scaling theory [36]. From a fit to the time-resolved transmission measurements for different $k l^{*}$, the transition to localization can be seen to take place at a value of $k l^{*} \simeq 4$.

acteristic length scales of absorption and localization. Therefore, we have to compare our static transmission measurements with diffusion theory including absorption, where the absorption length has been determined from the time-resolved measurements as discussed above. This is shown by the dashed curves in Fig. 5, where the shaded area indicates the error in the determination of the absorption length from the time-resolved measurements. As can be seen, this describes the classical sample very well, while still greatly overestimating the transmission in the localizing case, in contradiction with the data. A full description of the measurements is possible only when the localization length as determined from the timeof-flight measurements is also included. This yields the full curves in Fig. 5(b), which completely describe the data without any adjustable parameters over twelve decades in intensity.

\section{CONCLUSION}

In conclusion, we have shown that thick samples with very small values of $k l^{*}$ show increasing deviations from diffusive transport at long times (see Fig. 1). These deviations go together with the decrease of the localization length below the sample thickness, as obtained from a temporally varying diffusion coefficient (see Fig. 3). The onset of such a decreasing diffusion coefficient at long times can be associated with the transition to localization of waves, which takes place at $k l^{*} \simeq 4$ (see Fig. 4). In addition, these time-resolved measurements allow a determination of the absorption length, which does not show any systematic dependence on $k l^{*}$ (see Fig. 2), such that these deviations cannot be explained by effects of absorption. Moreover, other experimental effects, such as fluorescence, layering, a reduction in transport velocity, and background illumination can also be ruled out as the cause of the long-time tail in the time-of-flight distributions. Finally, a determination of the absorption length allows a quantification of static transmission measure-

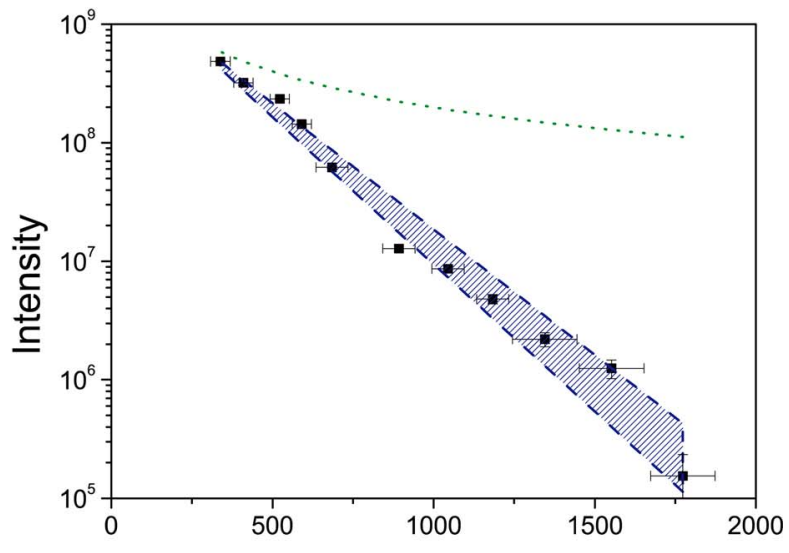

(a)

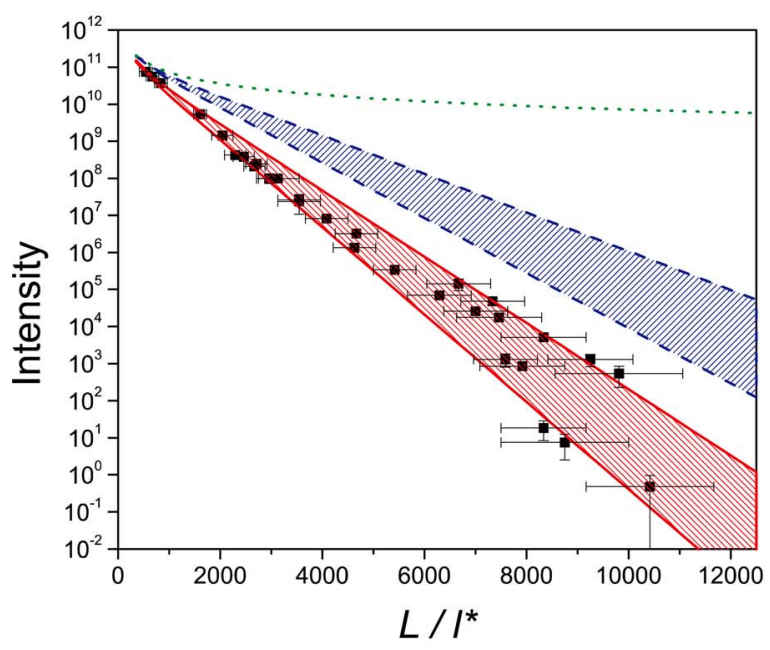

(b)

Fig. 5. (Color online) Static transmission of both a classical [(a), $\left.k l^{*}=25\right]$ and a localizing [(b), $\left.k l^{*}=2.5\right]$ sample as a function of thickness. While in both cases the decrease of the intensity is exponential and hence cannot be explained by pure classical diffusion (dotted curves), an unknown absorption could still explain the data. For $k l^{*}=25$ this is indeed the case, and including the measured absorption length from time-resolved measurements yields the dashed curves, which is in good agreement with the data. For the localizing sample however, this still contradicts the data. Only a description including the measured localization length as well gives an excellent fit to the data without a single adjustable parameter over 12 orders of magnitude. Note that there are no fitting parameters in any of the curves.

ments, where an exponential decrease faster than the one implied by absorption is observed (see Fig. 5). The discrepancy is well accounted for by introducing the localization length, also determined from time-resolved measurements [24]. Thus, the static transmission can be described without any adjustable parameters using localization theory over 12 orders of magnitude, which strongly supports the conclusion that we have indeed observed Anderson localization of visible light in three dimensions.

\section{REFERENCES}

1. P. W. Anderson, "Absence of diffusion in certain random lattices,” Phys. Rev. 109, 1492-1505 (1958). 
2. P. W. Anderson, "The question of classical localization: a theory of white paint?" Philos. Mag. B 52, 505-509 (1985).

3. S. John, "Electromagnetic absorption in a disordered medium near a photon mobility edge," Phys. Rev. Lett. 53, 2169-2172 (1984).

4. D. S. Wiersma, P. Bartolini, A. Lagendijk, and R. Righini, "Localization of light in a disordered medium," Nature (London) 390, 671-673 (1997).

5. J. M. Drake and A. Z. Genack, "Observation of nonclassical optical diffusion,” Phys. Rev. Lett. 63, 259-262 (1989).

6. F. Scheffold, R. Lenke, R. Tweer, and G. Maret, "Localization or classical diffusion of light?" Nature (London) 398, 206-207 (1999).

7. D. S. Wiersma, J. Gómez Rivas, P. Bartolini, A. Lagendijk, and R. Righini, reply to F. Scheffold, R. Lenke, R. Tweer, and G. Maret, "Localization or classical diffusion of light?" Nature (London) 398, 207 (1999).

8. A. A. Chabanov, M. Stoytchev, and A. Z. Genack, "Statistical signatures of photon localization," Nature (London) 404, 850-853 (2000).

9. M. P. van Albada, B. A. van Tiggelen, A. Lagendijk, and A. Tip, "Speed of propagation of classical waves in strongly scattering media," Phys. Rev. Lett. 66, 3132-3135 (1991).

10. G. H. Watson, P. A. Fleury, and S. L. McCall, "Searching for photon localization in the time domain," Phys. Rev. Lett. 58, 945-948 (1987).

11. P. M. Johnson, A. Imhof, B. P. J. Bret, J. G. Rivas, and A. Lagendijk, "Time resolved pulse propagation in a strongly scattering material," Phys. Rev. E 68, 016604 (1-9) (2003).

12. A. A. Chabanov, Z. Q. Zhang, and A. Z. Genack, "Breakdown of diffusion in dynamics of extended waves in mesoscopic media," Phys. Rev. Lett. 90, 203903 (1-4) (2003).

13. P. Sebbah, B. Hu, J. M. Klosner, and A. Z. Genack, "Extended quasimodes within nominally localized random waveguides," Phys. Rev. Lett. 96, 183902 (1-4) (2006).

14. S. K. Cheung, X. Zhang, Z. Q. Zhang, A. A. Chabanov, and A. Z. Genack, "Impact of weak localization in the time domain," Phys. Rev. Lett. 92, 173902 (1-4) (2004).

15. S. E. Skipetrov and B. A. van Tiggelen, "Dynamics of weakly localized waves," Phys. Rev. Lett. 92, 113901 (1-4) (2004).

16. A. F. Ioffe and A. R. Regel, "Non-crystalline, amorphous and liquid electronic semiconductors," Prog. Semicond. 4, 237-291 (1960)

17. M. Störzer, P. Gross, C. M. Aegerter, and G. Maret, "Observation of the critical regime near Anderson localization of light," Phys. Rev. Lett. 96, 063904 (1-4) (2006).

18. F. Scheffold, W. Härtl, G. Maret, and E. Matijević, "Observation of long-range correlations in temporal intensity fluctuations of light," Phys. Rev. B 56, 10942-10952 (1997).

19. F. Scheffold and G. Maret, "Universal conductance fluctuations of light," Phys. Rev. Lett. 81, 5800-5803 (1998).

20. S. E. Skipetrov and B. A. van Tiggelen, "Dynamics of Anderson localization in open 3D media," Phys. Rev. Lett. 96, 043902 (1-4) (2006)

21. S. E. Skipetrov, sergey.skipetrov@grenoble.cnrs.fr (personal communication, December 2005).

22. R. Berkovitz and M. Kaveh, "Propagation of waves through a slab near the Anderson transition: a local scaling approach," J. Chem. Soc. Dalton Trans. 2, 307-321 (1990).

23. R. Berkovitz and M. Kaveh, "Backscattering of light near the optical Anderson transition," Phys. Rev. B 36 , 9322-9325 (1987).

24. C. M. Aegerter, M. Störzer, and G. Maret, "Experimental determination of critical exponents in Anderson localization of light," Europhys. Lett. 75, 562-568 (2006).

25. P. E Wolf and G. Maret, "Weak localization and coherent backscattering of photons in disordered media," Phys. Rev. Lett. 55, 2696-2699 (1985).

26. M. P. van Albada and A. Lagendijk, "Observation of weak localization of light in a random medium," Phys. Rev. Lett. 55, 2692-2695 (1985)

27. E. Akkermans, P. E. Wolf, and R. Maynard, "Coherent backscattering of light by disordered media: analysis of the peak line shape," Phys. Rev. Lett. 56, 1471-1474 (1986).

28. J. C. M. Garnett, "Colours in metal glasses and in metallic films," Philos. Trans. R. Soc. London, Ser. A 203, 385-420 (1904).

29. J. X. Zhu, D. J. Pine, and D. A. Weitz, "Internal reflection of diffusive light in random media," Phys. Rev. A 44, 3948-3959 (1991)

30. K. Busch and C. M. Soukoulis, "Transport properties of random media: an energy-density CPA approach," Phys. Rev. B 54, 893-899 (1996).

31. P. Gross, "Coherent backscattering close to the transition to strong localization of light," Diploma Thesis, University of Konstanz (2005)

32. P. Gross, M. Störzer, S. Fiebig, M. Clausen, G. Maret, and C. M. Aegerter, "A precise method to determine the angular distribution of backscattered light to high angles," Rev. Sci. Instrum. 78, 033105 (1-6) (2007).

33. M. Störzer, C. M. Aegerter, and G. Maret, "Reduced transport velocity of multiply scattered light due to resonant scattering," Phys. Rev. E 73, 065602(R)1-4 (2006).

34. D. S. Wiersma, "Light in strongly scattering and amplifying random media," Ph.D thesis (University of Amsterdam, 1995).

35. R. Lenke, R. Tweer, and G. Maret, "Coherent backscattering and localization in a self-attracting random walk model," Eur. Phys. J. B 26, 235-240 (2002).

36. E. Abrahams, P. W. Anderson, D. Licciardello, and T. V. Ramakrishnan, "Scaling theory of localization: absence of quantum diffusion in two dimensions," Phys. Rev. Lett. 42, 673-676 (1979). 